\title{
ANÁLISIS DE LA TRANSICIÓN DE LA FÍSICA CLÁSICA A LA RELATIVISTA DESDE LA PERSPECTIVA DEL «CAMBIO CONCEPTUAL»
}

TOLEDO, B., ARRIASSECQ, I. y SANTOS, G. Departamento de Formación Docente. Facultad de Ciencias Exactas. Universidad Nacional del Centro de la Provincia de Buenos Aires. Pinto 399 (7000). Tandil, Argentina.

\section{SUMMARY}

Interviews about special relativity are realized with students of a Modern Physics course. The results of the interviews' analysis suggest that conceptual change doesn't mean a substitution of classic physics conceptions by the relativistic ones but that there is an interactive coexistence of both.

\section{INTRODUCCIÓN}

El presente trabajo pretende ser una contribución en el tema «cambio conceptual». Somos conscientes que actualmente esta denominación se ha generalizado, adquiriendo cierta ambigiedad inconveniente.Los resultados obtenidos nos permiten corroborar una opinión ya existente en la literatura (Lanciano, 1989; Moreira, 1994) pero que conviene aclarar cada vez más: el «cambio conceptual», en el sentido literal de la expresión, no existe; no sucede que una concepción existente se reemplaza por otra adquirida generalmente por instruccion formal. Ambas concepciones subsisten, incluso interligadas, en el sujeto que se mueve de un paradigma a otro. La búsqueda del «cambio conceptual» no es entonces lograr la sustitución de una concepción por otra, sino el encuentro de limitaciones y campo de validez de la "vieja» concepción y la aceptación de plausibilidad y eficiencia de la «nueva» concepción. En qué contexto y cuáles son los motivos o situaciones que actuarán como disparadores de una u otra es algo que todavía no está bien determinado. Existen situaciones típicas donde podemos predecir, pero nunca con certeza, a cuál acudirá. Por ejemplo, en un contexto de evaluación posible. mente recurrirá a la concepción «nueva» sobre la que es examinado, pero ante una situación nueva, en un contexto más abierto, ¿cuál usará?

La expresión cambio conceptual así interpretada pierde toda ambiguiedad. Probablemente esta expresion ahora no resulte adecuada; sería mejor habiar de evolución conceptual en un sentido toulminiano (Toulmin 1972). Pero la denominación cambio conceptual ya está instalada en ia literatura y resume la temática que abordamos a pesar de su significación literal. Preferimos mantenerla habiendo explicitado nuestra interpretación. 
Realizamos entrevistas sobre relatividad especial a los alumnos que recibieron instrucción sobre este tema. El contacto con el mismo significa un verdadero punto de inflexión en el conocimiento de la fúsica. Los alumnos se encuentran ante una situación nueva respecto a la física clásica y no pueden acudir a experiencias cotidianas para aceptar la plausibilidad de la teoria o para corroborar la eficiencia de la misma; deben basarse fundamentalmente en una abstracción reflexiva. Lo que puede haber de continuidad entre la física clásica y la relativista es menos relevante que aquello que las diferencia. Estamos ante un cambio conceptual y, si se realiza o no en cada estudiante, cómo y con qué profundidad, son ciertamente cuestiones pertinentes para nuestro estudio.

Con este trabajo también hemos podido responder algu. nas cuestiones que nos planteamos al preparar las entrevistas a los alumnos, tales como las consecuencias debidas a la ausencia de aportes epistemológicos en la instrucción sobre relatividad especial y en las que, en general, la primera asimilación del tema es superficial, de acuerdo con nuestra experiencia de que se aprende por aproximaciones sucesivas. Esta experiencia no está formulada de una manera ortodoxa, pero es compartida y encuentra su rigor, por ejemplo, en la teoría de la equilibración de Piaget (1975) con la idea de la espiral ascendente: Se ve la equilibración como una espiral que va ensanchándose desde una base muy estrecha hacia un bucle abierto al final (Vuyk, 1981, p. 190 de la edición castellana).

\section{MARCO TEÓRICO}

A mediados de la década de los setenta la comunidad docente de física y otras ciencias comenzó a considerar las ideas previas de los alumnos como variable importante en la enseñanza (Driver, 1973; Malgrange et al., 1973; Viennot, 1974). Ya no se consideró al alumno como terreno virgen donde podían inscribirse los conceptos formales de la ciencia sin competir con otros. Las ideas previas, concepciones intuitivas o conccpciones alternativas (respecto a las distintas denominaciones ver Toledo y Baquero, 1994) sobre diversos fenómenos interfieren con la enseñanza formal, máxime cuando no son correctas desde el punto de vista científico. En los últimos quince años, una gran cantidad de artículos han sido publicados sobre concepciones intuitivas y temas relacionados (Clement, 1982; Clement et al., 1989; Dibar Ure, 1991; Driver, 1986; Filocre, 1986; Gilbert y Swift, 1985; Gilbert y Zylberstajn, 1985; Guidoni, 1985; Gunstone, 1987; Halloun y Hestenes, 1985; Helm, 1980; McDermott, 1984; Millar, 1989; Moreira, 1990; Novak, 1983, 1987; Ogborn, 1985; Osborne et al., 1983; Pacca y Saraiva, 1989; Peduzzi, 1987; Pozo, 1987; Pozo et al., 1991; Queiroz y Azevedo, 1987; Saltiel y Viennot, 1985; Salvetti, 1986; Viennot, 1985).

Uno de los temas relacionados es el cambio conceptual. Una vez que se tomó conciencia de la importancia de las ideas previas, y dado que éstas pueden ser incorrectas, incompletas, dispersas o todo eso a la ve $z_{2}$ el esfuerzo en la instrucción se dirigio a sustituirlas por las concepciones científicas. La tarea se centró en buscar estrategias adecuadas para lograr el "cambio conceptual», quedando esta expresión ligada a la idea de sustitución; en realidad, la palabra cambio así parece indicarlo. En esta primera aproximación al problema se creía posible alcanzar tal cambio, que el alumno, una vez instruido en las concepciones vigentes de la ciencia, las aceptara y no volvicra a usar las previas que tenía, las cuales en el mejor de los casos, si eran coherentes y correctas desde el punto de vista científico, tenían un lugar en el nuevo paradigma. Pero esta primera aproximación simplificaba demasiado un problema que es complejo y, por lo tanto, no resultó adecuada. Con el auge de las concepciones intuitivas surgieron modelos para el cambio conceptual (Brown, 1992; Dykstra, 1991; Dykstra et al., 1992; Hashweh, 1986; Hewson, 1981; Hewson y Hewson, 1984, 1991; Hewson y Hennessey, 1991; Hewson y Thornley, 1989; Posner et al., 1982; Villani, 1992). Estos modelos no han alcanzado una validación empírica suficiente para suponer que constituyen una respuesta al problema. A pesar de ello, ch modelo desarrollado en los trabajos de Hewson (1981) y Posner y otros (1982) contiene elementos importantes, tales como plausibilidad e inteligibilidad de la nueva concepción presentada, que posiblemente seguirán teniendo vigencia en trabajos futuros donde más que en cambio conceptual (en el sentido de sustitución) se pensará en evolución conceptual, con interacción de las concepciones previas con las adquitidas por instruccion formal.

Actualmente aceptamos que las concepciones intuitivas coexisten con aquéllas trasmitidas por la instrucción formal, que esta coexistencia es interactiva y, como dijimos en la introducción, en la enseñanza no deberíamos tratar de cambiar una por otra sino de asumir la coexistencia trabajando en ese contexto, mostrando las ventajas de las concepciones científicas para explicar el mundo físico y no sólo situaciones aisladas. No se ha alcanzado una explicación detallada para la evolución conceptual. En una aproximación a este problema, Maloney y Siegler (1993) sugieren que la concepción, tanto la previa como la adquirida, no son únicas. Puede existir más de una concepción previa sobre el mismo fenómeno, también puede ser adquirida mús de una que compiten, y no meramente reemplazan, las previas. Es una aproximación interesante, ya que la cvidenciá empírica parece indicar que, en un sujeto, la concepción intuitiva sobre un hecho no siempre es una sola, y puede suceder que la concepción adquirida tampoco sea única. Esto último sería un resultado no buscado de la instrucción formal, que puede superarse, aunque no necesariamente, en un lapso de tiempo más extenso que el período de instrucción. Aqui tenemos implícita, otra vez, la noción de aprendizaje por aproximaciones sucesivas que mencionamos en la introducción.

En este trabajo consideramos la concepción previa, física clásica, y la que debieran adquirir, relatividad especial, como únicas. Sin negar la estructura fína que pueda tener cada una en el sentido sugerido por Maloney y Siegler, sólo indagamos sobre la coexistencia de ambas. 
Nos colocamos en un marco piagetiano, donde situamos las concepciones intuitivas en la primera construcción conceptual de causalidad que realiza el sujeto y, dentro de esa primera construcción, en el paso de la legalidad, u observancia de regularidades, a la explicación causal. Esta concepción puede no ser científicamente correcta, sea por una diferenciación no suficiente del sujeto, sea por una atribución no adecuada al objeto. Pero esa primera construcción no lo lleva a contradicción con lo observado; es decir, para el sujeto no hay error (Baquero et al., 1991). Además, en la teoría piagetiana, toda construcción de conocimiento se realiza por equilibración. Una concepción así construida es dif́́cilmente reemplazable, dado que no es probable una mera sustitución. El marco piagetiano nos proporciona elementos para entender los aspectos funcionales de la adquisición de conocimientos.

EI marco teórico se completa con una visión epistemológica. A mediados de nuestro siglo, la obra de Popper (1959), Lakatos (1978), Kuhn (1962) y Toulmin (1972) mostró la insuficiencia del análisis que realizó el neopositivismo para dar cuenta del conocimiento científico (Piaget y García, 1982). Adoptamos un enfoque touiminiano, ya que en él encontramos una mejor descripción de la evolución conceptual en el seno de una comunidad científica, con su noción de ecología intelectual a semejanza de la adaptación evolutiva biológica. Una comprensión de la evolución de los conceptos en una disciplina puede orientarnos para llegar a describir la evolución conceptual en un sujeto. La epistemología de Toulmin es decididamente evolucionaria, a diferencia de Kuhn, en el otro extremo, que adopta una descripción revolucionaria de los cambios. Si pensáramos el cambio conceptual como sustitución de una concepción por otra, la epistemología kuhniana sería adecuada, pero para nuestra hipótesis de coexistencia en interacción de ambas concepciones, la epistemología toulminiana resulta el marco adecuado. Esta coexistencia interactiva se desarrollará durante un tiempo, hasta que el sujeto adquiera profundidad en el conocimiento de la nueva concepción brindada por la instrucción formal, si ésta es exitosa. Entonces podrá distinguir los límites y la eficiencia de sus concepciones para usarlas adecuadamente. La más eficiente será más usada, lo que no implica la desaparición de la otra, sino más bien su relegamiento. Esta descripción evolutiva a grandes rasgos está basada en la noción de aprendizaje por aproximaciones sucesivas, coherente con ei cambio conceptual por evolución y no por sustitucion.

\section{METODOLOGÍA}

\section{A. Obtención de datos}

Se realizaron entrevistas clínicas con alumnos del curso de Física Moderna del año lectivo 1993. Se eligió la entrevista clínica por considerar que es un instrumento de indagación de mayor riqueza que una encuesta rígida. La materia Física Moderna, la cursan alumnos de $4^{\circ}$ año de la carrera Profesorado en Matemática y Física y alumnos de 3r. año de la carrera Licenciatura en Ciencias Físicas, ambas carreras de la Facultad de Ciencias Exactas. En 1993 cursaron ocho alumnos y respondieron aI pedido de entrevistas siete de ellos, dos varones de licenciatura y cinco mujeres de profesorado.

La preparación de las entrevistas y la elección del momento del año para realizarlas se hicieron tomando en cuenta un ensayo previo realizado en 1992. En efecto, guiado por las mismas ideas que hemos expuesto en las secciones anteriores, uno de los autores realiz6 entrevistas clínicas a los alumnos de Física Moderna de ese año. Relatividad especial es uno de los primeros temas de la materia citada. En aquel momento las entrevistas se llevaron a cabo a los pocos días de finalizar la instrucción formal sobre el tema. Las respuestas resultaron bastante escolarizadas; no nos parecieron suficientemente adecuadas para llegar a conclusiones válidas, por lo que decidimos repetirlas con los alumnos del año siguiente.

En 1993 se dejó pasar un lapso de cinco meses entre la instrucción y las entrevistas a fin de encontrar las nuevas ideas expresadas de una manera más personal. Se indagó sobre los contenidos específicos como en el año anterior, buscando los resultados más notables de la relatividad según la óptica del entrevistado y el nivel de comprensión que había alcanzado. Además se incorporó al libreto una parte epistemológica donde preguntamos sobre el origen de la teoría (en qué contexto surgió, cuáles fueron las causas de su aparición, las dificultades de su desarrollo, si compitió con otras teorias rivales, si fue una mera ampliación de la física clásica o una teoría distinta, etc.), dado que un conocimiento del origen y desarrollo de la teoría en la comunidad cientifica puede influir positivamente en el aprendizaje personal de la misma.

En la formulación final del libreto básico para entrevistas se explicitó como hipótesis de trabajo lo que se esperaba encontrar:

1) Que los nuevos conceptos de relatividad especial no desplazarían los anteriores de física clásica, sino que se unirían a ellos en una interacčión compleja. No se esperaba cambio conceptual en el sentido de sustitución sino en el sentido de coexistencia interactiva.

Como consecuencia inmediata se esperaba encontrar tambien:

2) Que el aprendizaje de contenidos específicos de relatividad especial sería más bien superficial, sin anclajes firmes que permitieran resolver situaciones fuera de aquéllas desarrolladas en la instrucción format.

Al incorporar la parte epistemológica se esperaba encontrar:

3) Que existiría escaso conocimiento sobre el origen de la teoría de la relatividad especial y esto impediría hacer una diferenciación respecto a la física clásica. 
Las entrevistas se realizaron en la forma usual, actuando uno como entrevistador y otro como observador. No tenían duración prefijada; se puede estimar un promedio de treinta minutos. A Ios alumnos se les aclaraba cuidadosamente que no nos interesaba examinar sus conocimientos en cuanto a su correccion. Se realizaron las siete entrevistas en un intervalo de tiempo menor de dos semanas. Se grabó el audio y luego se efectuaron las transcripciones con la confección de los respectivos protocolos.

\section{B. Análisis de las entrevistas}

En un primer análisis de las entrevistas se buscaron las ideas principales en cada una. Se hizo un resumen con tales ideas. Después se compararon los resúmenes y se volcaron los datos en una matriz: en las filas se colocaron los entrevistados, en las columnas las respuestas consideradas relevantes según la interpretación del analista. Se incorporó todo lo que se detectó en forma clara. Por ejemplo, cuando se preguntaba si existieron teorías rivales de la relatividad en la época de su surgimiento, algunos mencionaron la resistencia al cambio (en la transcripción de partes de protocolos que hacemos a continuación y a lo largo del artículo, Ias citas son breves, reducidas a su mínima expresión compatible con la captación de la idea, aunque puedan parecer descontextualizadas; en el punto B.2 mostraremos cómo llegamos a esta acotación):

«...aferrándose a la clásica...»

«...si él propuso esta teoría sería lógico que mucha gente se opusiera a aceptarla...»

«...pienso que no puede ser lo que se postula, que es algo imposible.»

En realidad, nosotros queríamos saber si conocían otras teorías que pretendieran explicar hechos que la clásica no explicaba, como la teoría de Lorentz. No habíamos previsto como respuesta posible el aferramiento a la clásica, pero la incorporamos. En este punto, aunque no conocieran teorías rivales, varios opinaron que existieron:

«Pienso que se deben haber hecho otros intentos.»

«...debe haber habido alguna otra porque siempre aparecen intentos de explicar. Seguro que la ha habido.»

«Yo entiendo que sí, porque siempre surgen, y más en una teoría como ésa, tiene que haber surgido alguien que dijo que nom.

"Yo pienso que podrían haber surgido».

La lectura de la matriz nos permitía concluir que se encontraba lo que habíamos esperado. Sin embargo, no nos parecía suficientemente objetivo el análisis. Esta inconformidad nos llevó a buscar otros métodos menos subjetivos para implementar un segundo análisis. Recu- rrimos a una herramienta de análisis que proviene del campo de las ciencias sociales y que no habíamos usado con anterioridad. Con esta nueva herramienta se reduce la información a unidades de significado que se pueden codificar, se establecen relaciones entre dichas unidades (agrupamientos, jerarquización, etc.). Una vez efectuada la etapa anterior se realiza un metaanálisis que es la comparación de las distintas entrevistas. Finalmente se puede intentar reconstruir las categorias subjetivas de los sujetos a investigar y con ello su visión del asunto (Huber, 1991, p. 11), que es el objetivo de nuestra indagación. Una ventaja importante de este análisis por unidades de significado es que puede llevarse a programas de computación, de hecho ya existen en circulación, por ejemplo AQUAD 3.0 (Huber, 1991). Pero lo que nos parece más importante es que nos brinda un marco de mayor objetividad a la vez que permite una validacion de nuestras hipótesis de trabajo explicitadas al planear el libreto básico.

Al realizar este segundo análisis de las entrevistas buscamos unidades de significado relevantes para nuestra tarea, que fueron codificadas. La identificación de unidades de significado en las transcripciones de las entrevistas pretende entresacar del discurso, de los textos de cada sujeto, las categorías que tengan importancia para reconstruir desde su propia perspectiva su mundo junto con su realidad circundante. La identificación y codificación de unidades de significado en los protocolos de las entrevistas fue realizada por cada autor separadamente; luego se cotejaron. No todos los autores habían propuesto las mismas; hubo ampliaciones, correcciones y modificaciones. Al final de las discusiones se llegó a un conjunto de 27 unidades de significado con sus códigos. (Están especificadas en el punto B.1 por orden alfabético de código.) Más que dar una definición pretendemos glosarlas mostrando las pautas que nos llevaron a identificar cada una, y agregamos un ejemplo. Nos hemos basado en el libro de Huber (1991), pero aplicamos nuestros propios criterios en la identificación de unidades de significados. Hay que destacar que en un mismo párrafo puede haber más de una unidad de significado. La longitud de la misma no está pređeterminada, la acotamos de manera que pueda ser interpretada por el analizador aun aistada del protocolo; a veces teníamos que mantener un párrafo entero; a veces bastaba una frase corta. Cuando se reduce a una sola palabra, en general afirmación o negación, tiene que ser interpretada unida a la pregunta previa del entrevistador. En el punto B.2 tratamos de mostrar con ejemplos, usando partes extensas de un protocolo, cómo procedimos para identificar y codificar unidades de significado.

\section{B.1. Unidades de significado y codigos asignados}

$A C L$ : Conocimientos sobre hechos, experimentales o teóricos, que no se podían explicar coherentemente dentro de la física clásica; anomalías en clásica.

Ejemplo: [...] como consecuencia de que no existra el éter. 
$C A U$ : Aceptación de un nuevo concepto por la autoridad científica de quien lo dice.

Ejemplo: Yo lo acepto porque, digamos; si los cientificos lo ven ast, que hacen estudios sobre esto, y hay teorias sobre esto, y ya están estudiando esto, lo acepto, pero no niego que no lo termino de entender.

$C E B$ : Conoce ejemplos de anomalías en física clásica y puede describirlos bien.

CEM: No conoce anomalías en física clásica o las conoce sin poder describirlas.

\section{Ejemplo: [...] la contracción de Fitzgerald-Lorentz.}

CER: Conoce anomalías en física clásica pero no puede describirlas correctamente.

Ejemplo: (...) encontrar un modelo que debia referirse a las ecuaciones de Maxwell en distintas situaciones.

CNC: Conoce ejemplos de corroboración de resultados de relatividad especial.

Ejemplo: I... el incremento de la masa al aumentar la velocidad, hay comprobaciones experimentales al acelerar particulas; se ve que cumplen con las predicciones.

CNP: Conoce correctamente los postulados de la relati* vidad especial.

Ejemplo: Bueno, los postulados son que la velocidad de la luz es la misma en cualquier referencial inercial y después que las leyes de la física se cumplen de la misma forma para todos los referenciales inerciales.

COT: Cotidiano, eventos o situaciones de la vida diaria.

Ejemplo: [...] para todo fenómeno que tiene que ver con lo que nosotros alcanzamos a ver, a visualizar digamos, con algo cotidiano.

CPO: Confusión entre postulados y resultados en relati* vidad especial.

Ejemplo: Me pareció novedoso que la luz se comportara [... I que la velocidad de la luz fuese constante.

\section{CTI: Contraintuitivo.}

Ejemplo: Creo que como los efectos se ven a alta velocidad, intuitivamente por ahi es dificil de aceptar.

CVC: Velocidad de la luz como constante.

Ejemplo: [...] supuestamente la velocidad de la luz es constante en cualquier sistema.

DCO: Dificuitad de comprensión, falta de plausibilidad.

Ejemplo: La dilatación del tiempo es como que todavía no la alcanzo a comprender.
DTE: la teoría especial de la relatividad y la física clásica son teorías distintas.

Ejemplo: No. La diferencia son los postulados.

$E B I:$ El concepto de espacio y el de longitud en relatividad especial resultan aceptables desde el punto de vista científico.

Ejemplo: $Y$, lo vería contraido.

ERE: Mención o uso del concepto de espacio o de longitud relativista.

Ejemplo: (...) si los tiempos eran distintos, también las longitudes eran; es decir, sería una dilatación de tiempo, habia una contracción de longitud, pero no sé lo que decian Fitzgerald y Lorentz.

LMC: Límite clásico de la teoría de la relatividad especial.

Ejemplo: Si para velocidades menores puedo trabajar con la relativista o la clásica, es la misma; y para mayores es la relativista.

MER: Mención o uso de los conceptos de masa, energía y masa en reposo en relatividad especial.

Ejemplo: [...] es la relación que establece entre la energia y la masa.

MST: Manifiesta tener alguna idea del surgimiento de una teoría científica.

Ejemplo: [...] llega un momento que, cuando la evolución de la ciencia ve que no hay respuestas a determinadas situaciones, tenés que plantear una nueva salida.

$M T E$ : La teoría especial de la relatividad y la física clásica son una misma teoría, aunque la relatividad es más general.

Ejemplo: Es una ampliación, no es distinta porque para límites clásicos, bueno, lo que decia yo hoy, esto se da para velocidades muy grandes, y para velocidades más chicas coinciden, coinciden las predicciones, las fórmulas.

NCC: No conoce ejemplos de corroboración de resultados de relatividad especial.

Ejemplo: $[. .$.$] no sé si eso será comprobable experimen-$ talmente.

OTE: Expresiones concernientes al contexto socio-his" tórico-científico en el cual surgió la teoría especial de la relatividad.

Ejemplo: [...] ha surgido Einstein con la respuesta a alguna situación, a un conflicto que en el momento no se podía resolver.

$R C A$ : No aceptación de la teoría especial de la relatividad por persistencia en el intento de explicación desde la física clásica. 
Ejemplo: [...] pienso que no puede ser lo que se postula, como que es algo imposible.

RIT: Conocimiento de otros intentos de explicar las anomalías de la física clásica; teorías rivales.

Ejemplo: Einstein se basó sobre cosas que [..] de Lorentz pero, bueno, no se si hizo primero él o no.

TBI: Concepto de tiempo aceptable en relatividad especial.

Ejemplo: [...] la dilatación del tiempo y la contracción de la longitud[...]

TRE: Mención o uso del concepto de tiempo relativista.

Ejemplo: [...] que el intervalo de tiempo se agrandara, se achicara, depende del sistema; pero, bueno, que sé yo, con el tiempo lo fui asimilando... es todo un punto de vista, no es que se agrande o se achique así... sino que todo depende de los sistemas que se toman.

$V B I$ : Corrección en el manejo de los contenidos de los códigos MER y VRS.

Ejemplo: [...] la parte de energía, y lo de las masas, que a medida que aumenta la velocidad, la masa cambia. $Y$ los ejemplos que vimos, por ejemplo, de las partículas en reposo y de las partículas que se mueven con velocidades comparables a la de la luz... como cambia su masa.

VRS: Mención o uso de los conceptos de velocidad, suma de velocidades y momento lineal relativista.

Ejemplo: (...) con respecto a otro sistema de referencia, el tiempo se dilata, entonces ese sistema debe estarse moviendo a velocidad de la luz, con velocidad comparable a la de la luz, para que valga la teoría relativista.

Los códigos no sólo simplifican las formulaciones individuales de cada entrevista, sino que a su vez representan los resultados interpretativos o explicativos. Es decir, la reducción de información o codificación es también un proceso interpretativo, que permitirá mejorar la objetividad y validez de los resultados. Establecidas las unidades de significado, se buscaron las relaciones entre ellas, o lo que es lo mismo, entre sus códigos. Así, en la parte epistemologica, se establece una cadena jerárquica tal que MST (surgimiento de una teoría) contiene OTE (contexto del surgimiento), éste contiene ACL (anomalías de la clásica) y éste contiene CEB, CER y CEM (conoce bien, regular o mal, ejemplos de anomalías); los tres últimos son distintos niveles de un mismo código. También MST contiene separadamente RIT (teorías rivales). Hemos dejado aparte RCA (resistencia al cambio) a pesar de su posible inclusión en RIT. Completando la parte epistemológica, MTE (relatividad y clásica una misma teoría) y DTE (relatividad y clásica distintas teorías) forman un subgrupo aparte. Podemos esquematizar estas relaciones así:

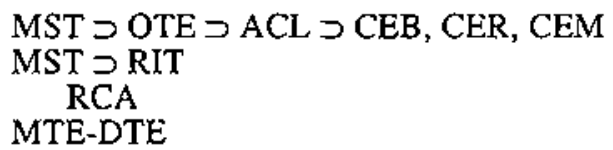

En la parte de contenidos específicos, forman el núcleo principal TRE (tiempo relativista), ERE (espacio y longitud relativistas), MER (masa y energía relativistas), VRS (velocidad, suma de velocidades y momento lineal relativistas) y LMC (límite clásico) acompañados, cuando corresponde, de los códigos terminados en $\mathbf{B l}$ que indican un manejo correcto de los contenidos. Fueron los que mejor mostraron las dificultades conceptuales de los alumnos. Una mención especial merece el código CAU (autoridad científica), que fue encontrado más de una vez en nuestro análisis, con repeticiones incluso en un mismo alumno. Nos parece una categoría importante para analizar la reacción de los alumnos ante una teoría nueva. Transcribimos algunos ejemplos representativos:

«Yo lo acepto porque, digamos, si los científicos lo ven así, que hacen estudios sobre esto, y hay teorías sobre esto, y ya están estudiando esto, lo acepto, pero no niego que no lo termino de entender.s

«[...] yo todavía no tengo una suficiente capacidad como para criticar esa teoría o ponerme a la altura de criticarla, tal vez, creería otra cosa."

«[...] aceptar, sí lo acepto porque me parece que sería una cosa necia de mi parte ponerme a discutir una cosa que todo el mundo la ha aceptado..s

\section{B.2. Identificación de unidades de significado}

En esta sección vamos a realizar la transcripción parcial pero con suficiente extensión de un protocolo de entrevista para mostrar cómo procedimos a identificar y acotar las unidades de significado. Las discusiones de los autores sobre la acotación fueron tantas como para la identificación. Al final triunfaron al mejor estilo popperiano aguéllas que mejor resistieron las refutaciones. Marcamos con < el comienzo de la unidad y con > el final; a continuación indicamos el código y denominación en forma breve. Si hay dudas por la presencia de dos o más unidades en un mismo párrafo, indicamos código al comienzo también. Usaremos notas si es necesario, intercaladas en la transcripción. El distinto tipo de letra evita confusiones. Todo lo que es protocolo está en cursiva. Para ahorrar espacio hacemos la transcripción a texto corrido, sin separar en columnas como es normal en los protocolos:

Entrevistador: Hace unos meses escucharon las clases sobre relatividad, ¿cuál es tu punto de vista?; ¿qué te parece?; ¿ cómo surge la teoría de la relatividad?

Nota: No se aclara relatividad especial porque es la única sobre la que fueron instruidos.

Alumna: Yo pienso que, según lo que fuimos estudiando nosotros, la teoria de la relatividad surgiría a fin de 
poder conocer fenómenos donde la velocidad es muy chica, no, muy grande, entonces < el conocimiento que conocemos habitualmente, de la vida diaria, se escaparía; o sea, los conocimientos que se estudian en relatividad son conocimientos que se escapan de lo que nosotros habitualmente manejamos. > COT(cotidiano) Porque según lo que fuimos estudiando, los fenómenos que fuimos viendo son con velocidades aproximadas a las de la luz. < Son velocidades que en la realidad no se dan > CTI(contraintuitivo) y entonces surge el inte. rrogante de cómo poder plantear determinadas leyes que se dan así cotidianamente con leyes donde... o sea, $<$ si esas leyes corresponden a otros fenómenos donde la velocidad no es como la que nosotros estamos acostum. brados a ver, entonces surge el interrogante de si hay transformación ( ; sic!) entre lo que se ve habitualmente y lo que surgiría en otra situación. $>$ MST (alguna idea del surgimiento de una teoría).

E: $X$ en el contexto en que surgió la teoría, ¿por qué te parece que surgió? ¿Fue una genialidad de Einstein aislada o había motivos?

A: No, lo que pasa es que < el estudio que fueron haciendo, digamos que sefue dando, sefue dando. No es algo que lo pensaron y dijeron; no, esto tiene que ser asi; no, digamos que elementalmente se fueron dando las cosas y entonces una respuesta llevó a la otra $>$ MST porque, por ejemplo, cuando se fue haciendo el estudio de las ondas, el estudio de las ondas electromagnéticas con Maxwell, bueno, ellos no sabían que la luz era una onda electromagnética, pero como ésta correspondia a las características que venían estudiando, entonces dijeron que era una onda eletromagnética, Y Einstein, por ejemplo, cuando elaborb lo de cuantización de la energía (Nota: posiblemente se refiere a la explicación del efecto fotoeléctrico) unos años después, lo elaboró a partir de algo experimental; o sea, < todas las cosas, digamos teóricas, fueron surgiendo a partir de la experiencia, de un dato empirico, al tratar de teorizar esas cosas que se dan empiricamente > OTE (contexto en el cual surgió la relatividad).

E: ¿Cuáles son los resultados de la teoría que te parecen más notables?

A: Nosotros deciamos que, cuando tomamos dos sistemas, uno con respecto al otro con velocidad constante o uniforme, si veiamos en un sistema, por ejemplo, la medición de una longitud, veíamos que $<$ la longitud aparece dilatada y que... no, aparece contraída > ERE (longitud relativista) y, si tomábamos < el tiempo aparecia dilatado > TRE (tiempo relativista) y TBI (concepto aceptablemente correcto) y eso a mí me asombró porque a mí me hubiera parecido que... pasaba exactamente lo mismo; o sea, de < hablar que algo se empequeñezca a mí me parecía asombroso porque todavía no lo veo, no lo veo visualmente. > CTI (contraintuitivo). Como... hablan que el tiempo es más largo, uno de los ejemplos era la teoría de los gemelos, y decian, bueno, que habia otros factores que intervenian, pero decian que si un astronauta se quedaba acá y el hermano gemelo iba a la luna o a algún lugar del espacio, entonces volvía más joven. Esas cosas que clásicamente -porque yo todavía tengo el pensamiento clásico- no..., me asombraron porque yo no las tenía presentes. $<$ Es como que me descolocaron, la dilatación y contracción de cosas que clásicamente no se dan, no se ven, digamos. >CTI (contraintuitivo).

\section{E: Algún otro resultado..}

A: Y... otra cosa; por ejemplo, que lo que se conservaba en ambos sistemas era CPO < la velocidad de la luz, cualquier sistema que tomara yo de referencia $\mathrm{CVC}<$ la velocidad de la luz iba a ser la misma $>>$ CPO (confusión entre postulados y resultados), CVC (velocidad de la luz como constante) < yo decía: ¿Cómo? unas cosas cambian y otras permanecen constantes; o sea, siempre hay alguna cosa que permanece constante en ambos sistemas. > DCO (dificultad de comprensión).

\section{E: Y con la energía, por ejemplo ¿qué pasa?}

A: $Y$ claro... cuando... lo de energía total y que no entendía era... o que no... < bajo velocidades comparables con la de la luz ya no era comparable la energía cinética como un medio de masa por velocidad al cuadrado sino que había que tomar en cuenta la energía que tenía en reposo $>$ MER (masa relativista) menos (isic!) la energía que tenía en movimiento y que < la masa que tenía en movimiento a esa velocidad comparable a la de la luz iba a ser más grande que la masa en reposo > MER, VBI (concepto aceptablemente correcto) y $<$ yo decía: 'Cómo!; o sea, no, como seguía pensando clásicamente esas cosas me parecía que no podian ser $>$ CTI (contraintuitivo) lo mismo que descubrieron que habia algo que no tenía masa pero que tenía energía, como es el fotón.

$\mathrm{E}$ : ¿Por qué dices que no puede ser, que piensas vos?

A: No digo que no puede ser, sino que, bajo el pensamiento mio, no lo hubiera imaginado; como todo lo que estudié en Física l y Física II son cosas clásicas, es difícil trasladar ahora a cosas que tienen que ver con relatividad o con temas posteriores, porque no puedo trasladar al clásico el tratar de ver algo que es relativista, tratar de transcribir que es igual; no es lo mismo, pero yo quiero hacer eso y no puedo. Entonces, bueno, esas cosas me cuestan. Lo mismo en cuanto a la energia, que < la energía puede transformarse en masa y que la masa puede transformarse en energia no lo veo; o sea, sí, la ecuación la tengo presente: energía igual a masa por c al cuadrado, pero no veo cómo se transforma energía en masa y viceversa $>$ MER (masa relativista) y DCO (dificultad de comprensión).

E: ¿Conoces alguna corroboración de estos resultados relativistas?

A: $<Y$ si, por ejemplo, cuando hablamos de la energía de enlace, el ejemplo que veíamos era la unión de partículas fundamentales, por ejemplo, el protón y el 
electrón (isicl) (Nota: se refiere el neutrón, para formar el deuterón). Si nosotros veíamos la energía en reposo de cada uno por separado y la sumábamos, nos daba una determinada energía pero, utilizando la formula de energia en reposo relativista, si veíamos la energla, en reposo de todo eso unido, nos daba un poco inferior; entonces donde se habia ido esa energía que por separado tenian y que unidas tenian menos, esa energía que faltaba era lo que, no sé, de alguna manera se habia desprendido para que se unieran, que era la misma que era necesaria para romperla. > MER, VBI (concepto aceptable) y CNC (conoce corroboración de resultados relativistas). (Nota: Mantenemos el párrafo entero porque acotaciones ensayadas rompen la ilación del relato. Es un ejemplo donde en el mismo lugar se identifican tres unidades de significado.)

E: ¿Y esos resultados te parecen plausibles? ¿Las cosas son asi?

A: Yo no digo que no son reales, sino que son dificiles de ver.

E: Pero vos... tu reticencia, ¿es porque no estás convencida?

A: < Yo lo acepto, digamos; si los científicos lo ven asi, que hacen estudios sobre esto, y hay teorias sobre esto, y ya están estudiando esto, lo acepto, pero no niego que no lo termino de entender > CAU (aceptación por autoridad científica).

Una vez completada la codificación de cada entrevista realizamos el metaanálisis o comparación de las entrevistas, a través de los códigos y relaciones ya establecidos. Las entrevistas muestran similitudes, las diferencias son más de grado que de naturaleza. En la búsqueda de resultados, es decir, al contrastar nuestras hipótesis de trabajo con las relaciones de los códigos, la frecuencia, Ia secuencia, la presencia o ausencia de los mismos que denotarían corroboración o no de dichas hipótesis, encontramos coherencia entre el metaanálisis y los análisis particulares.

\section{Resultados}

El análisis realizado de las entrevistas nos permite afirmar que se ha corroborado lo que esperábamos encontrar cuando enunciamos las hipótesis de trabajo. Mientras que para dos de esas hipótesis es suficiente trabajar sólo con las unidades de significado, para la de coexistencia interactiva entre ambas concepciones, clásica y relativista, tenemos que regresar al análisis directo de Ios textos con el riesgo de perder objetividad. Sucede que nuestras entrevistas estaban centradas en indagaciones sobre relatividad; no disponemos entonces de unidades de significado sobre clásica equiparables a las usadas -recordemos que Ias entrevistas fueron realizadas antes que se decidiera el uso de una herramienta de análisis más poderosa. En trabajos futuros, este hecho puede subsanarse.

\section{C.1. Coexistencia interactiva}

El mero hecho de que existan componentes clásicos en las respuestas a cuestiones relativistas ya es un indicio de la coexistencia postulada. Vamos a examinar estas situaciones a través de tres ejemplos de distintos entrevistados (en la transcripción de estos ejemplos hemos omitido la codificación):

\section{Ejemplo 1}

Entrevistador: ¿Qué otro resultado te ha llamado la atención?

Alumna: Quizá también lo de suma de velocidades, lo que se espera clásicamente y...

\section{E: A ver explícame un poco más.}

A: ...que clásicamente, por ejemplo, si un autombvil se mueve a determinada velocidad, a $30 \mathrm{~km} / \mathrm{h}$, y pasa uno en la misma dirección y se mueve a $80 \mathrm{~km} / \mathrm{h}$, la velocidad relativa de uno respecto al otro es $50 \mathrm{~km} /$ h. En cambio, para velocidades comparables a la de la luz, si un auto va a determinada velocidad y envía un destello de luz, la velocidad total del sistema va a ser la de la luz más la del auto, lo que se esperaría clásicamente. Sin embargo relativisticamente, la velocidad de la luz es siempre c.

\section{$\mathrm{E}:$ O sea que se suman distinto las velocidades.}

A: Es decir, no tendría sentido sumar porque son velocidades no comparables, una es mucho más chica que la otra.

(Nota: Si en un principio parecía captar la diferencia entre ambas teorías, luego permanece en la concepción clásica cuando reduce todo a una cuestión de órdenes de magnitud. En realidad, no distingue dos teorías, considera que es una con la novedad de algún añadido.)

\section{Ejemplo 2}

E: Bueno, respecto a los resultados de la relatividad, ¿cuál te pareció más notable?

A: El tiempo, cómo manejar el tiempo... la dilatación del tiempo, es como que te choca, no te das cuenta, no alcanzás a comprender cómo puede ser que varíe el tiempo de esa manera. La parte del espacio es como que la veo más. La dilatación del tiempo es como que todavia no la alcanzo a comprender.

$\mathrm{E}:$ ¿Que entendés vos por la parte del espacio? A ver, por ejemplo, si un objeto se mueve muy rápido y yo estoy en reposo...

\section{A: Lo veo más chico.}

E: ¿Y eso no te causa problemas? 
A: No, es como si... me da la impresión de que, si yo lo veo pasar, es algo propio, es muy casera mi idea, como que una cosa que va tan rápido puede ser que la vea más chica... pero, el tiempo, no me doy cuenta como es que se ve así, que se alarga el tiempo.

Nota: Su idea «casera» es consecuencia de tratar de interpretar un resultado relativista dentro de un marco clásico; toma Ia contracción como algo real. Otra vez, aquí, no hay delimitación de teorías, se mezclan elementos de ambas como si fueran una.

Nota: Aunque creemos que no tiene incidencia en el presente análisis, es conveniente señalar otra posible fuente de obsiáculos para este caso: la dificultad que tienen los alumnos para ubicarse en distintos sistemas de referencia; eso se nota en mecánica clásica, en el tema de fuerzas inerciales.

\section{Ejemplo 3}

E: Bueno, ¿qué pasa con la suma de velocidades en relatividad? ¿Conoces?

A: No me acuerdo mucho. Sé que hay una fórmula relacionada con la velocidad de la luz.

E: ¿Cuál sería la diferencia, no sé si lo tendrás presente, respecto al papel de la velocidad de la luz en relatividad y en clásica?

A: Bueno, sería más o menos lo que dije hoy: si yo tomo velocidad comparable con la de la luz... interviene la teoría relativista, sino interviene la clásica.

Nota: Aquí hay de alguna manera una delimitación de las teorías, pero con campos de existencia disjuntos; si la velocidad del sistema físico no es comparable a la de la luz, no es lícito aplicar relatividad.

E: Por ejemplo, en clásica, si tengo una fuente que emite luz y esa fuente se mueve, su velocidad se suma, vectorialmente, a la de la luz. ¿En relatividad se hace lo mismo?

\section{A: Sí.}

E: ¿Vos tienes presente los postulados de la relatividad?

A: Sí, la velocidad de la luz es constante en cualquier sistema y con respecto a cualquier sistema de referencia.

E: Entonces, decir que, si la fuente se mueve, le sumo la velocidad de la fuente a la de la luz, itambién lo hago en relatividad?

\section{A: $S i$.}

E: Si a c le sumo otra velocidad, tendría una velocidad mayor a c. ¿Eso está admitido por los postulados de Einstein?
A: No.
E: Entonces, ¿cómo es la situación?

A: (No contesta.)

E: ¿Puedo componer la velocidad de la luz con otras velocidades desde el punto de vista de la relatividad?
A: $N o$.
E: Pero me parece que lo primero que me dijiste es que si.

A: Claro, pero no, ahora como usted dijo, no puede ser, no puede darse una velocidad mayor que la de la luz.

E: Quiero entender bien lo que vos crees, porque lo primero que me dijiste, y lo dijiste convencida, es que, si la fuente de luz se mueve, a c le tengo que sumar la velocidad de la fuente.

\section{A: Claro.}

$\mathrm{E}$ : Suponete que la fuente se mueve en esta dirección (se indica). En esa dirección, ¿la velocidad de la luz es co es $\mathrm{c}+\mathrm{v}$ ?
A: No, es c.
E: Y en clásica, ¿cómo es?

\section{A: También es c.}

Nota: La delimitación que se había insinuado más arriba, aquí ha desaparecido totalmente; la mezcla es muy fuerte, y resulta en contradicciones insuperables mientras no distinga las teorías.

Hay otros ejemplos menos representativos que los mostrados. Creemos que todos ellos corroboran nuestra conjetura de coexistencia. Podría pensarse que los conceptos de ambas teorías coexisten separadamente, pero como consideran la relatividad especial como un apéndice de la física clásica, los conceptos están mezclados. $\mathrm{Si}$ bien uno de los alumnos está más cercano que otros al "paradigma relativista», ninguno ha llegado a él. Nos parece una mejor descripción hablar de evolución conceptual, pasando por etapas intermedias que aún no estamos en condiciones de describir, en Iugar de cambio conceptual en el sentido de sustitución.

\section{C.2. Aprendizaje superficial}

Esta hipótesis puede ser corroborada usando las unidades de significado codificadas. En efecto, cada vez que aparecen los códigos MER (masa o energía relativistas) o VRS (velocidad, suma de velocidades y momento lineal relativistas) no siempre van acompañados de VBI (concepto aceptable), es mayor el número de veces que aparecen solos que las veces que aparecen con VBI. Lo mismo sucede con ERE (espacio o longitud relativista) 
NOVAK, J. D. (1983). Proceeding of the International Seminar Misconception in Science and Mathematics, en HELM, H, y NOVAK, J.D. (eds.). Nueva York: Cornell University, Ithaca.

NOVAK, J.D. (ed.) (1987). Proceeding of the Second International Seminar: Misconceptions and Educational Strategies in Science and Mathematics. Nueva York: CornelI University, Ithaca.

OGBORN, J. (1985). Understanding student's understandings. European Journal of Science Education, 7, pp. 141-150.

OSBORNE, R.J., BELL, B.F. y GILBERT, J.K. (1983). Science teaching and children's view of the world. European Journal of Science Education, 5, pp. 1-14.

PACCA, J.L.A, y SARAIVA, J.A.F. (1989), Causalidad y operaciones en la interpretación de las concepciones espontáneas. Enseñanza de las Ciencias, 7, pp. 266-270.

PEDUZZI, L.G. (1987). Soluçao de problemas e conceitos intuitivos, Caderno Caterinense de Ensino de Fisica, 4, pp. 17-24.

PIAGET, J. (1950). Introduction a l'epistémologie genetique,II. La pensee physique. París: PUF. Trad. cast., 1975, Buenos Aires: Paidós.

PIAGET, J. (1975). L'equilibration des structures cognitives. Problème central du developpement. París: PUF. Trad. cast. 1978, Madrid: Siglo XXI.

PIAGET, J. y GARCIA, R. (1971). Les explications causales. París: PUF. Trad. cast. 1973, Barcelona: Barral.

PIAGET, J. y GARCÍA, R. (1982). Psicogénesis e historia de la ciencia. México: Siglo XXI.

POPPER, K.R. (1959). The logic of scientific discovery. Londres: Hutchinson.

POSNER, G. J., STRIKE, K. A., HEWSON, P.W. y GERTZOG, W.A. (1982), Accommodation of a scientific conception: toward a theory of conceptual change. Science Education, $66\{2\rangle$, pp. 211-227.

POZO, J.I. (1987). Aprendizaje de la ciencia y pensamiento causal. Madrid: Editorial Visor.
POZO, J.I., SANZ, A., GÓMEZ CRESPO, M. A. y LIMÓN, M. (1991). Las ideas de Ios alumnos sobre ciencia: una interpretación desde la psicología cognitiva. Enseñanza de las Ciencias, 9(1), pp. 83- 94.

QUEIROZ, G. y AZEVEDO,C.A.(1987). A ciencia alternativa do senso comum e o treinamento de professores. Caderno Caterinense de Ensino de Física, 4, pp. 7-16.

SALTIEL, E. y VIENNOT, L. (1985). ¿Qué aprendemos de las semejanzas entre las ideas históricas y el razonamiento espontáneo de los estudiantes? Enseñanza de las Ciencias, 3 , pp. $137-144$.

SALVETTI, A.P. (1986). Conceitos espontaneosem Física. Os problemas inerentes a uma definiçao. Revista de Ensino de Fisica, 8(1), pp. $49-58$

TOLEDO, B. y BAQUERO, R. (1994). ¿Diversos nombres o diversos problemas? Las denominaciones usuales de las concepciones intuitivas. Revista de Enseñanza de la Física, 7(1), pp. 3-10.

TOULMIN, S. (1972). Human understanding. I: The collective use and evolution of concepts. Princenton University Press. Trad. cast. 1977. Madrid: Alianza Editoriat.

VIENNOT, L. (1974). Sens physique e raisonnement formel en dynamique elementaire. Bulletin de la Societe Française de Physique-Encart Pedagogique, 2, pp. 35-46.

VIENNOT, L. (1985), Analysing student's reasoning in science: a pragmatic view of theoretical problems. European Journal of Science Education, 7, pp. 151-162

VILLANI, A. (1992). Conceptual change in science and science education. Science Education, 76(2), pp. 223-237.

VUYK, R. (1981). Overview and critique of Piaget's Genetic Epistemology, 1965-1980, 2 vol. Londres: Academic Press. Trad. cast. 1984. Madrid: Alianza Editorial.

VYGOTSKY, L. S. (1934). Myshlénie i rech. Moscú. Trad. cast. 1992. Pensamiento y lenguaje. Buenos Aires: Ediciones Fausto-Editorial La Pléyade. 